\title{
Distribution and survival of primary sarcoma in Korea: A single center analysis of 2017 cases
}

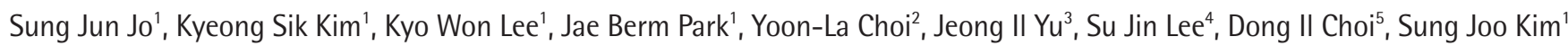
Departments of 'Surgery, ${ }^{2}$ Pathology, ${ }^{3}$ Radiation Oncology, ${ }^{4}$ Medicine, and ${ }^{5}$ Diagnostic Radiology, Samsung Medical Center, Sungkyunkwan University School of Medicine, Seoul, Korea

Purpose: Distribution and survival of sarcoma in Korea are not well described, after the changing of sarcoma classification on 2013. The researchers investigated the distribution and survival in single center 2017 cases of sarcoma.

Methods: Patients with primary sarcoma, who underwent surgery, were investigated. All cases were collected during a 20 year period (19952015) from Samsung Medical Center in Korea. Histopathologic types were classified by World Health Organization (WHO) classification (2013). And overall survival rates were analyzed.

Results: Between 1995 and 2015, 2017 patients were collected. The most frequent type of sarcoma was gastrointestinal tumor (15\%), followed by liposarcoma (12\%), leiomyosarcoma (9\%), dermatofibrosarcoma (6\%), giant cell sarcoma (6\%). The most common primary site of sarcoma was the intra-abdominal area (45\%, including visceral area). Extremities accounted for $26 \%$ of all cases. Sixteen percent of sarcoma were located in retroperitoneal area. The overall sunvival rate was 70.4\% (median follow-up time, 36.8 months; range, 0.1-261.3 months). The best prognosis was dermatofibrosarcoma (100\%, 5-year survival rate). The worst prognosis was angiosarcoma (39.3\%). Survival analysis by the primary site demonstrated favor prognosis in extremities than head \& neck, chest lesion.

Conclusion: The researchers reported Korean sarcoma characteristics with using the new WHO classification.

Keywords: Sarcoma, Epidemiology, Korea

\section{INTRODUCTION}

Sarcomas were mesenchymal originated tumors, arising in connective tissue. These were a very rare incidence of overall human malignant tumors, accounting for less than $1 \%$ of all new cancer diagnoses [1]. This heterogeneous malignancy tumor consists of 70 different histologic subtypes [2].

Histopathologic classification of sarcoma has changed considerably since 1978 [3]. The World Health Organization (WHO) published new sarcoma classification, representing updated consensus, in 2013. According to this, gastrointestinal tumor (GIST) has in-

Received: Feb 26, 2018 Accepted: May 21, 2018

Correspondence to: Sung Joo Kim

Department of Surgery, Samsung Medical Center, Sungkyunkwan

University School of Medicine, 81 Irwon-ro, Gangnam-gu, Seoul 06351,

Korea

Tel: +82-2-3410-3476, Fax: +82-2-3410-0040

E-mail: kmhyj111@gmail.com

Copyright @ C Korean Society of Surgical Oncology

This is an Open Access article distributed under the terms of the Creative Commons Attribution Non-Commercial License (http://creativecommons.org/licenses/by-nc/4.0) which permits unrestricted non-commercial use, distribution, and reproduction in any medium, provided the original work is properly cited. cluded in sarcoma and many cases of malignant fibrous histiocytoma (MFH) were classified as specific sarcoma [4]. Many previous reports about the epidemiology of sarcoma were not based on new classification. Only a few studies described the characteristics of sarcoma, and prognosis with new classification.

In Korea, one study reported epidemiology and prognosis of sarcoma. It was based on a previous classification with low volume of data [5]. Therefore, the aim of the study is to analyze the distribution, characteristics, prognosis of sarcoma based on new classification with a retrospective review of single center 2,017 cases.

\section{METHODS}

Data were retrospectively collected from a database of Samsung Medical Hospital, Seoul, Korea, between January 1995 and December 2015. Patients who underwent surgery for primary sarcoma were investigated. Data collected included age, gender, date of surgery, histological type, primary site, completeness of resection, 5-year survival, and overall survival (OS).

Primary sarcoma was defined as a tumor that had not been treated previously, and no evidence of metastasis. Tumors which 
underwent second surgery due to positive cancer cell on resection margin on first surgery at other hospital were included in this study.

Histologic types were reviewed according to the WHO classification [4]. In the GIST, both high risk group (National Institutes of Health consensus criteria, 2002) and malignant types were included. Tumors were classified according to 15 histologic groups: GIST, liposarcoma, leiomyosarcoma, dermatofibrosarcoma, giant cell tumor, chondrosarcoma, undifferentiated sarcoma, osteosarcoma, MFH, primitive neuroectodermal tumor (PNET), rhabdomyosarcoma, angiosarcoma, malignant peripheral nerve sheath tumor (MPNST), synovial sarcoma, others (solitary fibrous tumor, myxosarcoma, fibrosarcoma, epithelioid sarcoma, clear cell sarcoma, alveolar soft-part sarcoma, and so on). Liposarcomas were subdivided into five subtypes: well-differentiated, myxoid, dedifferentiated, pleomorphic, not otherwise specified (NOS). The following cases were excluded, for the reasons explained in parentheses: mesothelioma (mesothelial tumors of the pleura), neuroblastoma (tumors of the autonomic nervous system), mesenchymomas (an entity not clearly defined), carcinosarcoma (tumors that is mixture of carcinoma), ganglioneuroblastoma (tumors of the autonomic nervous system). Primary sites were divided into six groups: head \& neck, chest, abdominal cavity, upper extremities, lower extremities, others (genital organ, pelvic bone, and vascular lesion). The abdominal cavity was subdivided into three groups: intra-abdominal, retroperitoneal, abdominal wall. The researchers illustrated primary sites and proportion of histological subtypes on one figure (Fig. 1).

Completeness of resection was categorized as clear (R0), microscopically positive (R1), grossly positive (R2). Gross resection status was determined with operating record documented by an operating surgeon. Margin status was investigated based on a pathologic report. A clear margin indicated there was no tumor on resection margin microscopically. A microscopically positive margin indicated microscopically extension of cancer cell regardless of distance from the tumor margin. In addition, cases of undetermined margin status in pathologic report with grossly total resection were considered as $\mathrm{R} 1$ resection.

Overall survivorships were calculated from the date of surgery to the last known date of follow-up or the date of death. Date of death was identified from the linkage of Korean Statistical Information Service. Disease specific death was not investigated. Based on 15 histologic subtypes of sarcomas and six primary sites, sur-

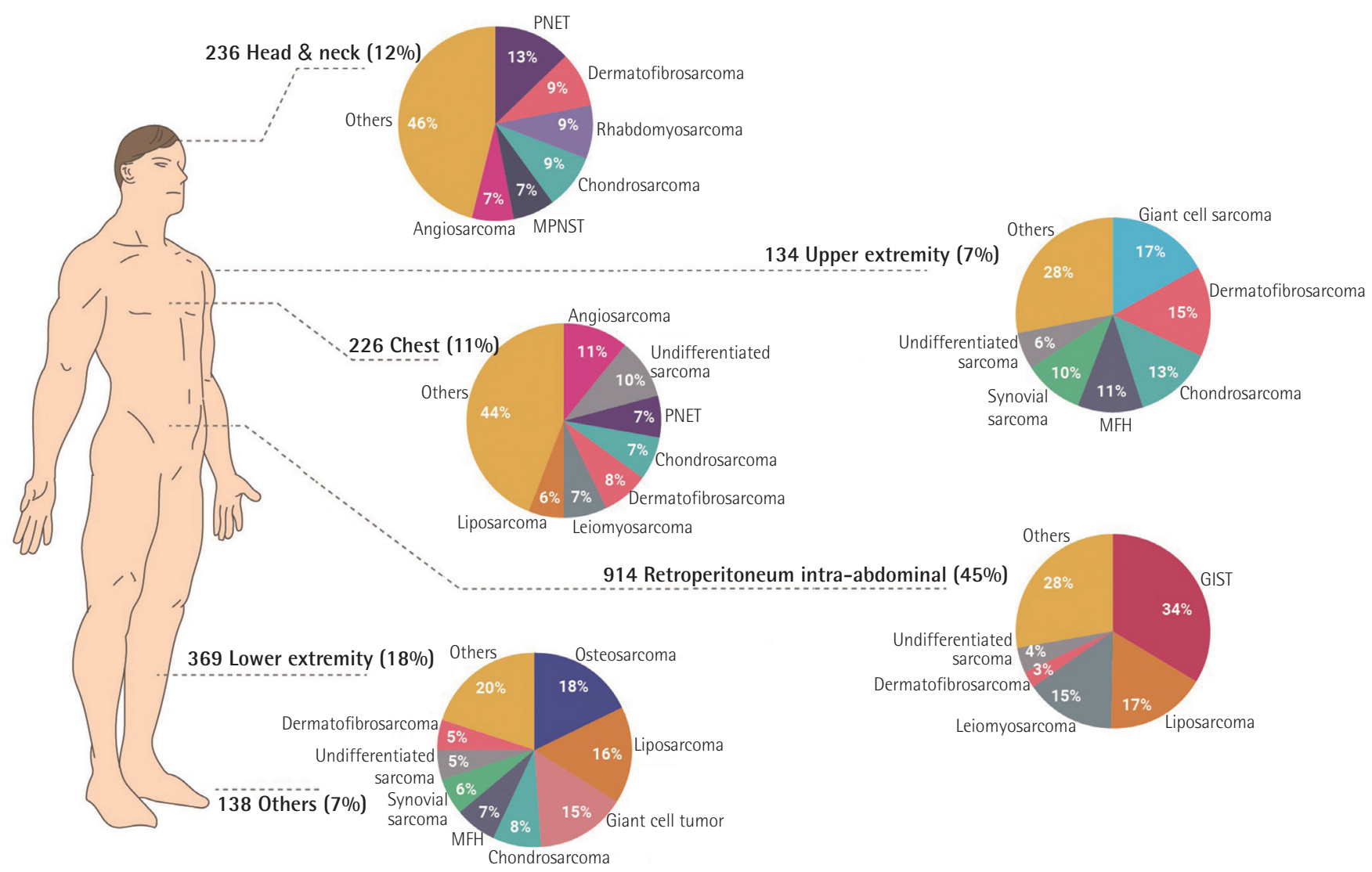

Fig. 1. Subtype distribution according to histologic types, primary sites. PNET, primitive neuroectodermal tumor; MPNST, malignant peripheral nerve sheath tumor; MFH, malignant fibrous histiocytoma; GIST, gastrointestinal tumor. 
vival rates were calculated for each types and sites. Estimation of survival was calculated by the Kaplan-Meier method and survivorship analysis, being assessed by the log-rank test.

Survival graphs were illustrated and analyzed by grouping similar origin of sarcomas together. Rhabdomyosarcoma, chondrosarcoma, osteosarcoma, synovial sarcoma and leiomyosarcoma were grouped in musculoskeletal group PNET, MPNST and angiosarcoma were in neurovascular group. The other subtypes were grouped in unclassified group including GIST and liposarcoma. On the other hand, survival graphs by primary sites in all kinds of sarcoma were illustrated all together. All analysis was done using SPSS version 23.0 (IBM Corp., Armonk, NY, USA). Statistical significance was defined as $\mathrm{P}<0.05$.

\section{RESULTS}

\section{Subtype distribution}

Two thousand, one hundred and fifty-eight patients, who underwent surgery for primary sarcoma, were investigated. One hundred and forty-one patients were excluded because of palliative surgery. The median age was 48 years (range, $0-91$ years; mean, 45.6 years). The ratio of male to female was 1.04:1. The seventy-one percent $(n=1,425)$ of patients underwent grossly complete excision, whereas 157 patients (8\%) had grossly margin positive. Among all patients, the most common histologic type was GIST (15\%), followed by liposarcoma (12\%), leiomyosarcoma (9\%), dermatofibrosarcoma (6\%), giant cell sarcoma (6\%) (Table 1).

The most common primary site was the abdominal cavity (45\%), followed by the lower extremity (18\%), head \& neck (12\%), chest (11\%), others (7\%), and upper extremity (7\%). Even when dividing the abdominal cavity into three regions: intra-abdominal (27\%), retroperitoneal (16\%), abdominal wall (2\%), the intra-abdominal was still the most common site of sarcoma. In the head \& neck region, PNET (13\%) was the most common histologic type. Five types of sarcoma (dermatofibrosarcoma [9\%], rhabdomyosarcoma [9\%], chondrosarcoma [9\%], MPNST [7\%], angiosarcoma [7\%]) accounted for half of the rest. For the thoracic region (chest), the most common histologies were angiosarcoma (11\%), undifferentiated sarcoma (10\%), followed by dermatofibrosarcoma $(8 \%)$, primitive neuroectodermal sarcoma $(7 \%)$, chondrosarcoma (7\%), leiomyosarcoma (7\%), and liposarcoma (6\%). In the abdominal cavity, GIST (34\%) was the most common histologic type and liposarcoma (17\%) was the second most common type. For extremity sarcoma, the giant cell tumor was the most common subtype on the upper extremity (17\%) but be on the third place on lower extremity (15\%). Osteosarcoma (18\%), liposarcoma (16\%) were the most common types on the lower extremity (Fig. 1).
Table 1. Demographic of the patients $(n=2,017)$

\begin{tabular}{|c|c|}
\hline Variable & Value \\
\hline \multicolumn{2}{|l|}{ Sex } \\
\hline Male & $1,032(51)$ \\
\hline Female & $985(49)$ \\
\hline Age (yr) & $48(0-91)$ \\
\hline \multicolumn{2}{|l|}{ Location } \\
\hline Head \& neck & $236(12)$ \\
\hline Chest & $226(11)$ \\
\hline Upper extremity & $134(7)$ \\
\hline Abdominal cavity & $914(45)$ \\
\hline Intra-abdominal & $550(27)$ \\
\hline Retroperitoneal & $315(16)$ \\
\hline Abdominal wall & $49(2)$ \\
\hline Lower extremity & $369(18)$ \\
\hline Others & $138(7)$ \\
\hline \multicolumn{2}{|l|}{ Histology } \\
\hline Gastrointestinal tumor & $311(15)$ \\
\hline Liposarcoma & $252(12)$ \\
\hline Leiomyosarcoma & $187(9)$ \\
\hline Dermatofibrosarcoma & $124(6)$ \\
\hline Giant cell sarcoma & $113(6)$ \\
\hline Chondrosarcoma & $106(5)$ \\
\hline Undifferentiated sarcoma & $100(5)$ \\
\hline Osteosarcoma & $95(5)$ \\
\hline Malignant fibrous histiocytoma & $80(4)$ \\
\hline Primitive neuroectodermal tumor & $78(4)$ \\
\hline Rhabdomyosarcoma & $63(3)$ \\
\hline Angiosarcoma & $61(3)$ \\
\hline Malignant peripheral nerve sheath tumor & $55(3)$ \\
\hline Synovial sarcoma & $55(3)$ \\
\hline Others & $337(17)$ \\
\hline \multicolumn{2}{|l|}{ Completeness of resection } \\
\hline Ro & $1,425(71)$ \\
\hline $\mathrm{R} 1$ & $435(21)$ \\
\hline $\mathrm{R} 2$ & $157(8)$ \\
\hline
\end{tabular}

Values are presented number (\%) or median (range).

The overall primary site distribution of each histologic subtypes was displayed on Fig. 2. Most of GIST were in abdominal cavity $(85 \%)$. Retroperitoneal area was accounted for $13 \%$. Only $1 \%$ of GIST were in thoracic region. All GIST cases of thoracic region were occurred on esophagus. In liposarcoma, majority of lesion were in retroperitoneal area (50\%). Lower extremity was accounted for $23 \%$. The head \& neck was the most common primary site of PNET (38\%).

\section{Survival}

The overall sarcoma survival rate was $70.4 \%$, with a median follow-up time of 36.8 months (range, $0.1-261.3$ months). The best prognosis subtype was the dermatofibrosarcoma (100\%) and followed by GIST (90\%). Whereas angiosarcoma (39.3\%) demon- 
- Head \& neck $\square$ Thoracic $\square$ Intra-abdominal $\square$ Retroperitoneal $\square$ Upper extremity $\square$ Lower extremity

Others (including abdominal wall)

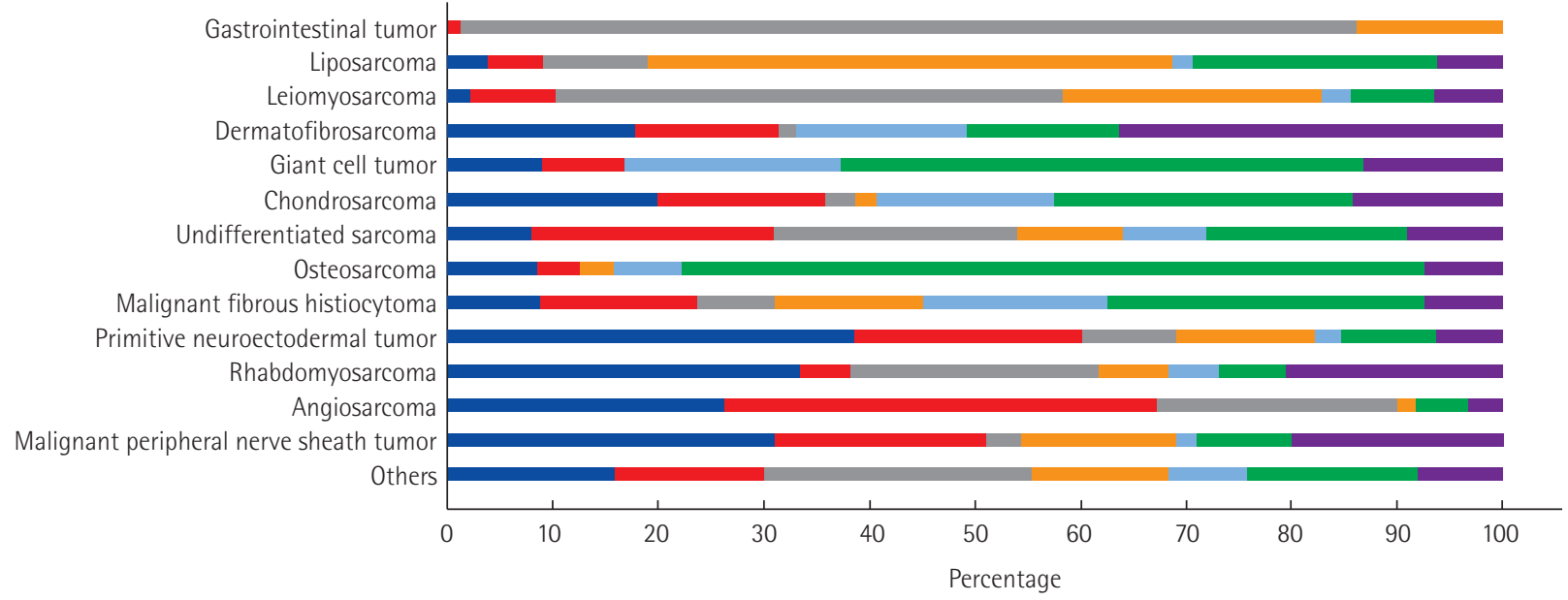

Fig. 2. Primary site distribution of histologic subtypes.
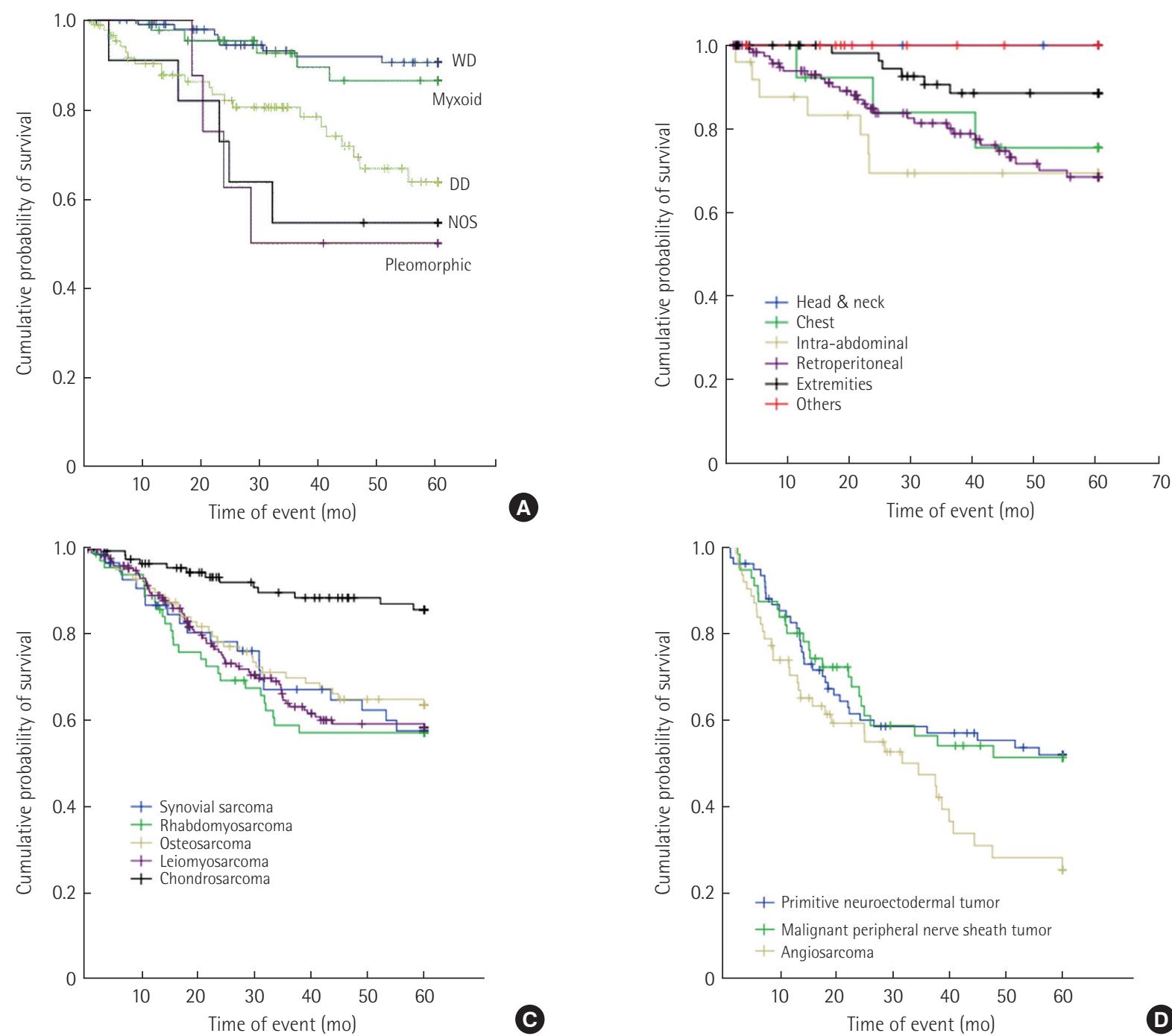

B

Fig. 3. Survival outcome according to subtypes. (A) Five-year overall survival (OS) of liposarcoma for each subtypes, (B) 5-year OS of liposarcoma for each primary site, (C) 5-year OS of musculoskeletal group, (D) 5-year OS of neurovascular group. WD, well differentiated; DD, dedifferentiated; NOS, not otherwise specified. 

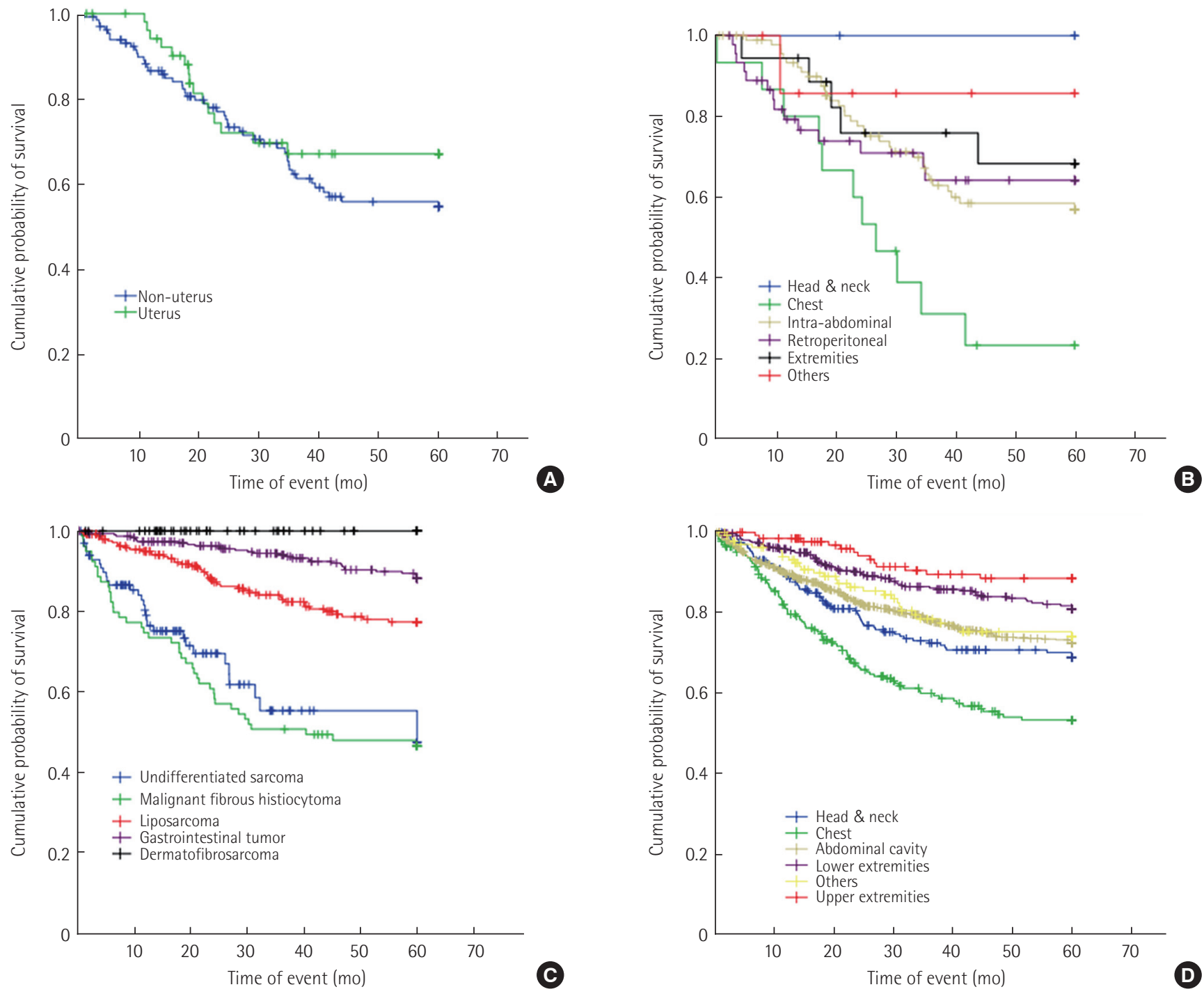

Fig. 4. Survival outcome according to subtypes. (A) Five-year overall survival (OS) of leiomyosarcoma in uterus, non-uterus group, (B) 5-year OS of leiomyosarcoma for each primary site, (C) 5-year OS of others group, (D) 5-year OS of primary site.

strated the worst prognosis.

In the liposarcoma group, survivorship was differed depending on subtypes, ranging from $54.5 \%$ (pleomorphic) to $91.8 \%$ (welldifferentiated). When survivorships were compared each subtype with liposarcoma, NOS, well-differentiated liposarcoma (93.5\%, $\mathrm{P}<0.0001)$ and myxoid liposarcoma $(88.6 \%, \mathrm{P}<0.009)$ demonstrated a better survivorship than liposarcoma NOS (55.6\%). The 5-year survivorships of each subtype are demonstrated in Fig. 3A. Survivorship comparison by primary sites was higher in extremities $(90.5 \%)$ than retroperitoneal $(77.6 \%, \mathrm{P}<0.009)$, intra-peritoneal $(72.0 \%, \mathrm{P}<0.015)$ (Fig. 3B).

In the musculoskeletal group, chondrosarcoma (87.7\%) demonstrated a better prognosis than the other four subtypes (synovial sarcoma [63.6\%], rhabdomyosarcoma [58.7\%], osteosarcoma
[66.3\%], leiomyosarcoma [65.2\%], P < 0.001). Except chondrosarcoma, the other subtypes were demonstrated a similar progressive decrease in survivorship graph (Fig. 3C). For the neuromuscular group, angiosarcoma (39.3\%) had a poor prognosis in 5-year survival than MPNST (56.4\%, P <0.019), PNET (54.7\%, P <0.037). All the three types were demonstrated similar survival graph until 30 months. However, after 30 months of follow-up, angiosarcoma demonstrated a more significant decrease (Fig. 3D). Especially in leiomyosarcoma, uterus group (72.2\%) was demonstrated favor survivorship than non-uterus group (62.4), however it was statistically not significant $(\mathrm{P}<0.254)$ (Fig. 4A, B).

In others group, there was no death in dermatofibrosarcoma patients during 5-year follow-up period. On the comparison with liposarcoma $(82.2 \%)$ histologic subtype, undifferentiated sarcoma 
(69.0\%), MFH (46.3\%) were shown poor prognosis, whereas dermatofibrosarcoma (100\%), GIST (90.0\%) were better $(\mathrm{P}<0.001)$ (Fig. 4C).

In the comparison of survival rates according to primary sites, the upper extremities (90.2\%), and the lower extremities (83.7\%) demonstrated less progressive decrease in survivorship graphs than others (79\%). Abdominal cavity (76.2\%) and head \& neck (72.9\%) were shown similar curves with others group, but chest group (59.9\%) were shown more progressive decrease in survivorship graph. However only upper extremities $(\mathrm{P}<0.004)$, chest region $(\mathrm{P}<0.001)$ were statistically significant (Fig. $4 \mathrm{D})$.

\section{DISCUSSION}

The aim of this study was to estimate histologic distribution, distribution of location, survival rates of sarcoma in the Korea. In addition, this is the first study to show a distribution of sarcoma, including GIST in Korea. In the data, the most common histologic type was GIST, accounting $15 \%$ of all. Hui previously reported liposarcoma was the most common [6]. This difference was due to the fact that our institute had only an abdominal sarcoma center without an emphasis on limb sarcoma. In addition, the high incidence of GIST in Korea (16-22 per million) might have affected the underestimation of liposarcoma [7,8]. GIST, liposarcoma and leiomyosarcoma were the most common histologic subtypes. This finding was concurrent with previous other reports $[5,9,10]$. In addition, the most common place of sarcoma was the abdominal cavity, which was more than sum of upper and lower extremities. Previous reports were reported the extremities as the common place. This difference was due to change of sarcoma classification, especially including GIST [4,5,11]. Distribution of histologic types in other lesions (head \& neck, thoracic, extremities) was not concurred with any previous study $[5,6,12]$. It is not clear why the research data demonstrated a different distribution, especially a low proportion of MFH. However, the exception of metastatic cases and changes in diagnostic criteria of MFH might be an influence in result [3]. Distribution of primary site by the histologic types was concurred with other reports $[6,8,13]$. For instance, most of GIST occurred in the stomach and small intestine. The retroperitoneum and lower extremities were the most common place in liposarcoma. One third of PNET and rhabdomyosarcoma were diagnosed on the head \& neck.

In the present study, 5-year OS of GIST (90\%) was demonstrated higher than previous reports (50\%-65\%) [14,15]. It is unclear why our study demonstrated higher survivorship because this study did not investigate characteristics (mitotic index, size) of GIST. Therefore, further research of this findings will be needed.
Regarding to histologic distribution of liposarcoma in this study, well differentiated and de-differentiated type were the most common types. This is concurrent with previous report of $\mathrm{Oh}$ et al. [16]. Which analyzed 213 patients' survival for liposarcoma regarding to histologic subtypes and primary sites. In addition, the 5 -year OS according to histologic types was also similar with this research data, favorable 5-year OS in well-differentiated liposarcoma than dedifferentiated liposarcoma and pleomorphic liposarcoma. Previous studies classified inguinal/genitalia as a new group of primary sites in liposarcoma, whereas this study classified inguinal/genitalia, and head \& neck as other group altogether. The reason for this classification was that the most of head \& neck liposarcoma were occurred in scalp, cheek, skin of neck. Therefore, in this study, the others group demonstrated better survival than retroperitoneal group without death in 5-years.

Chondrosarcoma was found to demonstrate a significant higher 5-year OS $(\mathrm{P}<0.001)$ than other subtypes in musculoskeletal group (synovial sarcoma, rhabdomyosarcoma, osteosarcoma, leiomyosarcoma). Stiller et al. [17] also reported the favor prognosis of chondrosarcoma (76.7\%) than osteosarcoma (53.9\%) [18]. However, previous reports analyzed survivorship by classifying sarcoma as soft tissue group (leiomyosarcoma, rhabdomyosarcoma, synovial sarcoma) and bone sarcoma group (osteosarcoma, chondrosarcoma) [17-19]. There was no recent report of survivorship analysis between chondrosarcoma and rhabdomyosarcoma, leiomyosarcoma.

Leiomyosarcoma demonstrated $65.2 \%$ in 5-year OS in present study, which is similar with other reports $(64.2 \%-65.7 \%)[20,21]$. In subgroup analysis between uterus group $(72.2 \%)$ with non-uterus group (62.4\%) in leiomyosarcoma, demonstrated favor survivorship in uterus group than non-uterus group without statistical significance in present study. Lamm et al. [22] that analyzed $95 \mathrm{pa}-$ tients of leiomyosarcoma (50 non-uterus leiomyosarcoma, 45 uterus leiomyosarcoma) also reported the better prognosis in uterus group than non-uterus group ( $82.6 \%$ vs. $41.2 \%, \mathrm{P}<0.006$ ).

The most of study did not analyze soft tissue sarcoma and bone sarcoma together because of its different subtypes, different natural history and different response to therapy [16-18]. However, this study was conducted distribution and survivorships analysis together. Therefore, It will be need more specific survivorship analysis in future study by subgrouping as soft tissue sarcoma, bone tissue sarcoma, adult (over 18 age) sarcoma, child sarcoma (under 18 age) etc.

There are some limitations in this study. First, it was a retrospective analysis. Second, a pathologic slide review was not conducted. Therefore, there can be misdiagnosed cases due to changing classifications. In addition, as mentioned above, because of characteris- 
tics in the study institution (having only abdominal sarcoma center without Limb sarcoma center). Selection bias also exists.

These data demonstrated subtypes and survival rate for sarcoma patients in South Korea by new classification of WHO (2013). The distribution of sarcoma seems to be similar with pervious report. Whereas survival rates were higher than previous reports especially in GIST. The reason of this finding was not clarified; further investigation is needed to confirm this finding.

\section{CONFLICT OF INTEREST}

No potential conflict of interest relevant to this article was reported.

\section{REFERENCES}

1. Ressing M, Wardelmann E, Hohenberger P, Jakob J, Kasper B, Emrich K, et al. Strengthening health data on a rare and heterogeneous disease: sarcoma incidence and histological subtypes in Germany. BMC Public Health 2018;18:235.

2. Fairweather M, Wang J, Jo VY, Baldini EH, Bertagnolli MM, Raut $\mathrm{CP}$. Incidence and adverse prognostic implications of histopathologic organ invasion in primary retroperitoneal sarcoma. J Am Coll Surg 2017;224:876-83.

3. Toro JR, Travis LB, Wu HJ, Zhu K, Fletcher CD, Devesa SS. Incidence patterns of soft tissue sarcomas, regardless of primary site, in the surveillance, epidemiology and end results program, 19782001: an analysis of 26,758 cases. Int J Cancer 2006;119:2922-30.

4. Jo VY, Doyle LA. Refinements in sarcoma classification in the current 2013 World Health Organization classification of tumours of soft tissue and bone. Surg Oncol Clin N Am 2016;25:621-43.

5. Choi KU, Kang HY, Koo H, Kwon MS, Kim DH, Kim MJ, et al. Type and incidence of soft tissue sarcomas in Korea: 2001-2007. Korean J Pathol 2011;45:557-63.

6. Hui JY. Epidemiology and etiology of sarcomas. Surg Clin North Am 2016;96:901-14.

7. Cho MY, Sohn JH, Kim JM, Kim KM, Park YS, Kim WH, et al. Current trends in the epidemiological and pathological characteristics of gastrointestinal stromal tumors in Korea, 2003-2004. J Korean Med Sci 2010;25:853-62.

8. Soreide K, Sandvik OM, Soreide JA, Giljaca V, Jureckova A, Bulusu VR. Global epidemiology of gastrointestinal stromal tumours (GIST): a systematic review of population-based cohort studies. Cancer Epidemiol 2016;40:39-46.
9. Corey RM, Swett K, Ward WG. Epidemiology and survivorship of soft tissue sarcomas in adults: a national cancer database report. Cancer Med 2014;3:1404-15.

10. Honore C, Meeus P, Stoeckle E, Bonvalot S. Soft tissue sarcoma in France in 2015: epidemiology, classification and organization of clinical care. J Visc Surg 2015;152:223-30.

11. Coindre JM, Terrier P, Guillou L, Le Doussal V, Collin F, Ranchere $\mathrm{D}$, et al. Predictive value of grade for metastasis development in the main histologic types of adult soft tissue sarcomas: a study of 1240 patients from the French Federation of Cancer Centers Sarcoma Group. Cancer 2001;91:1914-26.

12. Sturgis EM, Potter BO. Sarcomas of the head and neck region. Curr Opin Oncol 2003;15:239-52.

13. Ferrari A, Sultan I, Huang TT, Rodriguez-Galindo C, Shehadeh A, Meazza C, et al. Soft tissue sarcoma across the age spectrum: a population-based study from the Surveillance Epidemiology and End Results database. Pediatr Blood Cancer 2011;57:943-9.

14. Lai EC, Lau SH, Lau WY. Current management of gastrointestinal stromal tumors: a comprehensive review. Int J Surg 2012;10:33440.

15. Li J, Zhang H, Chen Z, Su K. Clinico-pathological characteristics and prognostic factors of gastrointestinal stromal tumors among a Chinese population. Int J Clin Exp Pathol 2015;8:15969-76.

16. Oh YJ, Yi SY, Kim KH, Cho YJ, Beum SH, Lee YH, et al. Prognostic model to predict survival outcome for curatively resected liposarcoma: a multi-institutional experience. J Cancer 2016;7:1174-80.

17. Stiller CA, Trama A, Serraino D, Rossi S, Navarro C, Chirlaque MD, et al. Descriptive epidemiology of sarcomas in Europe: report from the RARECARE project. Eur J Cancer 2013;49:684-95.

18. Hodel S, Seeli F, Fuchs B. Demographic analysis of patients with osteosarcoma, chonddrosarcoma, Ewing's sarcoma from one sarcoma center in Switzerland. Praxis (Bern 1994) 2015;104:673-80.

19. Skubitz KM, D’Adamo DR. Sarcoma. Mayo Clin Proc 2007;82:140932.

20. Cui RR, Wright JD, Hou JY. Uterine leiomyosarcoma: a review of recent advances in molecular biology, clinical management and outcome. BJOG 2017;124:1028-37.

21. Yu T, Kim HJ, Wu HG, Ha SW, Song YS, Park NH, et al. Outcome analysis in patients with uterine sarcoma. Radiat Oncol J 2015;33:2935 .

22. Lamm W, Natter C, Schur S, Kostler WJ, Reinthaller A, Krainer M, et al. Distinctive outcome in patients with non-uterine and uterine leiomyosarcoma. BMC Cancer 2014;14:981. 